\title{
Financial Inclusion for Self Help Groups
}

\author{
Dr. ThotaNagaraju \\ Associate Professor, PG. Dept. of Commerce, \\ SSMRV College, Jaya Nager, Bangalore
}

\author{
Dr. TalluriSreekrishna \\ Sr. Associate Professor, Dept. of Management \\ Sciences, R.V.R \& J.C. College of Engineering, \\ Chowdavaram, Guntur
}

\section{ABSTRACT}

Rangarajan's Committee on Financial inclusion may be defined as the process of ensuring access to financial services and timely and adequate credit where needed by vulnerable groups such as weaker sections and low income groups at an affordable cost.Rangarajan's Committee on Financial inclusion may be defined as the process of ensuring access to financial services and timely and adequate credit where needed by vulnerable groups such as weaker sections and low income groups at an affordable cost." With a view to reach financial inclusion to the poor and rural mass in this country the government of India identified the financial inclusion is a strategy to achieve the inclusive growth provided and it is supported by various factors like real initiatives from Banks and Financial Institutions, technological development, financial literacy and so on. Amongst various measures to fight this menace, micro finance practices in India seem to provide a solution. The SHG - Bank Linkage programme had proved its efficiency as a main stream programme for Banking and emerged as one of the need based policies and programmes to cater the neglected groups of society such as woman, poor and deprived sections of rural areas. Several studies made by national and international experts on micro finance have found the SHG profitable, viable and as a successful tool or social empowerment and also no bank has reported any NPA under the SHG Bank linkage. The beautiful advantages of the programme are on time repayment of loans to banks, reduction in transaction cost to the poor and to the banks, door - step savings and credit facilities to the poor and exploitation of the untapped business potential in rural India. The programmed started as an outreach programme has in fact, achieved more than mere provision of thrift and credit facilities to the poor women. The government of India and State governments can play vital roles in encouraging SHGs. They should formulate and redefine their strategies and policies such a way to stress on extensive awareness campaign, skill development and training programmes, co ordination between banks and SHGs, effective flow of credit need for strong follow - up in those states where it is yet at nascent stage. It is also necessary to develop a sound and transparent regulatory structure for micro finance institutions for healthy growth of the sector along with supportive refinance and legal.

\section{FINANCIAL INCLUSION FOR SELF HELP GROUPS}

The poverty in India is wide spread as well as deep rooted and continues to be one of the biggest policy concerns. Amongst various measures to fight this menace, Microfinance practices in India seem to provide a solution. The Task Force on supportive policy and regulatory Frame work for Microfinance constituted by NABARD defined microfinance as "the provision of thrift, saving, credit and financial services and products of very small amount to the poor in rural, semi-urban and urban areas for enabling them to raise their income levels and improving their standard of living." The Banking system in India witnessed unprecedented growth and achieved phenomenon out reach. Apart from the existing banking network, with a view to developing a supplementary credit delivery system i.e. cost effective and user friendly for both banks and the 
poor, micro finance initiatives were encouraged in India. These initiatives have been centered around two models i.e. the SHG - Bank linkage programme \& the Micro Finance Institutions (MFI's) model. Women, who number 495.7 million according to 2001 census, represent $48.3 \%$ and 933 females per thousand males. It reveals that the decline of women number in total population of the country. Moreover nearly $1.5 \%$ of women have their own property. About $11 \%$ women are in employment and mere 5\% have been participating in business. The present paper is an attempt to study the Micro Finance through SHG's.

\section{The Concept of Self Help Group's (SHGs):}

Micro financing is a new method to meet the credit requirement in rural areas. Since the bank borrowing requires collateral and the deprived class does not have any type of such collaterals, the success of Bangladesh Grameena Banks attracted the attention of Indian Policy makers towards the Micro finance and micro credit, which are the new entrants in realm of present rural financing.

The Self Help Group (SHG) bank linkage model has emerged as the most dominant model of Micro Finance delivery in India. A Self Help Group (SHG) is a registered or unregistered group of micro entrepreneur's with homogeneous social and economic background, voluntarily coming together with an average size of about 15 individuals. They come together for addressing their common problems. They are encouraged to make voluntary thrift on a regular basis. They use this pooled resource to make small interest bearing loans to their members. The process helps them imbibe the essentials of financial intermediation including prioritization of needs, setting terms \& conditions and account keeping. This gradually builds financial discipline in all of them. The Self Help Group (SHG) members begin to appreciate the fact that resources are limited and have a cost. Once the groups show this mature financial behavior, banks are encouraged to make loans to the Self Help Group (SHG) in certain multiples of the accumulated savings of the Self Help Group (SHG). The bank loans are given against group dynamics without any collateral and at market interest rates. Since the group's own accumulated savings are part and parcel of the aggregate loans made by the groups to their members, peer pressure ensures timely repayments.

\section{Self Help Group's (SHGs) - Bank Linkage Programme:}

The SHG - Bank linkage programme was started as an action research project in 1989. The pilot project was launched by NABARD in 1992 with policy support from Reserve Bank of India. The pilot project was designed as a partnership model $b / w$ three agencies, via the SHG's, Banks and Non Governmental Agencies (NGO's). The SHG's were expected to facilitate collective decision making by the poor and provide door step banking, the banks as wholesalers of credit, were to provide the resources, while the NGO's were to act as agencies to organize the poor, build their capacities and facilitate the process of empowering them.

Table: 1- Overall Progress under Micro-Finance during the last three years ( in crore)

\begin{tabular}{|c|c|c|c|c|c|c|c|}
\hline \multirow{2}{*}{\multicolumn{2}{|c|}{ Particulars }} & \multicolumn{2}{|c|}{ 2012-13 } & \multicolumn{2}{|c|}{ 2013-14 } & \multicolumn{2}{|c|}{ 20014-15 } \\
\hline & & $\begin{array}{l}\text { No. } \\
\text { of } \\
\text { SHGs }\end{array}$ & Amount & $\begin{array}{l}\text { No. of } \\
\text { SHGs }\end{array}$ & Amount & $\begin{array}{l}\text { No. of } \\
\text { SHGs }\end{array}$ & Amount \\
\hline \multicolumn{8}{|c|}{ A) SHGs Bank linkage Model } \\
\hline \multirow{2}{*}{$\begin{array}{c}\text { Savings } \\
\text { of SHGs } \\
\text { with } \\
\text { Bank as } \\
\text { on 31 } \\
\text { March }\end{array}$} & $\begin{array}{l}\text { Total } \\
\text { SHGs }\end{array}$ & $\begin{array}{c}50097 \\
94\end{array}$ & 3785.39 & $\begin{array}{c}6121147 \\
(22.2)\end{array}$ & $\begin{array}{c}5545.62 \\
(46.5)\end{array}$ & $\begin{array}{c}6953250 \\
(13.6)\end{array}$ & $\begin{array}{c}6198.71 \\
(11.8)\end{array}$ \\
\hline & $\begin{array}{l}\text { Out of } \\
\text { which } \\
\text { SGSY }\end{array}$ & $\begin{array}{c}12030 \\
70\end{array}$ & 809.51 & $\begin{array}{c}1505581 \\
(25.1)\end{array}$ & $\begin{array}{c}1563.38 \\
(93.1)\end{array}$ & $\begin{array}{c}1693910 \\
(12.5)\end{array}$ & $\begin{array}{c}1292.62 \\
(17.3)\end{array}$ \\
\hline
\end{tabular}


International Journal of Trend in Scientific Research and Development (IJTSRD) ISSN: 2456-6470

\begin{tabular}{|c|c|c|c|c|c|c|c|}
\hline \multirow{2}{*}{$\begin{array}{c}\text { Bank } \\
\text { loans } \\
\text { disburse } \\
\text { d to } \\
\text { SGHs } \\
\text { during } \\
\text { the year }\end{array}$} & $\begin{array}{l}\text { Total } \\
\text { SHGs }\end{array}$ & $\begin{array}{c}12277 \\
70\end{array}$ & 8849.26 & $\begin{array}{c}1609586 \\
(31.1)\end{array}$ & $\begin{array}{c}12253.51 \\
(38.5)\end{array}$ & $\begin{array}{c}1586822 \\
(1.4)\end{array}$ & $\begin{array}{c}14453.30 \\
(17.9)\end{array}$ \\
\hline & $\begin{array}{l}\text { Out of } \\
\text { which } \\
\text { SGSY }\end{array}$ & $\begin{array}{c}24664 \\
9\end{array}$ & 1857.74 & $\begin{array}{c}264653 \\
(7.3)\end{array}$ & $\begin{array}{c}2015.22 \\
\quad(8.5)\end{array}$ & $\begin{array}{c}267403 \\
(1.0)\end{array}$ & $\begin{array}{c}2198.00 \\
(9.1)\end{array}$ \\
\hline \multirow{2}{*}{$\begin{array}{c}\text { Bank } \\
\text { loans } \\
\text { outstand } \\
\text { ing with } \\
\text { SGHs as } \\
\text { on } 31 \\
\text { March }\end{array}$} & $\begin{array}{l}\text { Total } \\
\text { SHGs }\end{array}$ & $\begin{array}{c}36259 \\
41\end{array}$ & 16999.91 & $\begin{array}{c}4224338 \\
(16.5)\end{array}$ & $\begin{array}{c}22679.84 \\
(33.4)\end{array}$ & $\begin{array}{c}4851356 \\
(14.8)\end{array}$ & $\begin{array}{c}28038.28 \\
(23.6)\end{array}$ \\
\hline & $\begin{array}{l}\text { Out of } \\
\text { which } \\
\text { SGSY }\end{array}$ & $\begin{array}{c}91697 \\
8\end{array}$ & 4816.87 & $\begin{array}{c}976887 \\
(6.5)\end{array}$ & $\begin{array}{c}5861.72 \\
(21.7)\end{array}$ & $\begin{array}{c}1245394 \\
(27.5)\end{array}$ & $\begin{array}{c}6251.08 \\
(6.6)\end{array}$ \\
\hline \multicolumn{8}{|c|}{ B) MFI - Bank linkage Model } \\
\hline & & \multicolumn{2}{|c|}{$2012-13$} & \multicolumn{2}{|c|}{$2013-14$} & \multicolumn{2}{|c|}{$2014-15$} \\
\hline 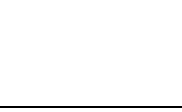 & 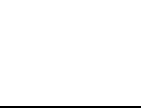 & $\begin{array}{c}\text { No. of } \\
\text { MFI }\end{array}$ & Amount & No. of MFI & Amount & No. of MFI & Amount \\
\hline \multicolumn{2}{|c|}{$\begin{array}{c}\text { Bank loans } \\
\text { disbursed to } \\
\text { MFIs during the } \\
\text { year }\end{array}$} & 518 & 1970.15 & $\begin{array}{c}581 \\
(12.2)\end{array}$ & $\begin{array}{c}3732.33 \\
(89.4)\end{array}$ & $\begin{array}{c}691 \\
(18.9)\end{array}$ & $\begin{array}{c}8062.74 \\
(116.0)\end{array}$ \\
\hline \multicolumn{2}{|c|}{$\begin{array}{c}\text { Bank loans } \\
\text { outstanding with } \\
\text { MFIs as on } 31 \\
\text { March }\end{array}$} & 1109 & 2748.84 & $\begin{array}{c}1915 \\
(72.7)\end{array}$ & $\begin{array}{c}5009.09 \\
(82.8)\end{array}$ & $\begin{array}{c}691 \\
(21.0)\end{array}$ & $\begin{array}{c}8062.74 \\
(102.6)\end{array}$ \\
\hline
\end{tabular}

The performance of SHG - Bank Linkage Programme continued to be the predominant micro finance model in India. (During 2012- 13, 686,408 new SHGs were credit linked with banks, taking the cumulative number of SHGs credit linked to 2.92 million. In addition, 457,410 existing SHGs received repeat finance during the year.

Table 2: Microfinance - Savings of SHGs with Banks Region-wise and Southern States as on 31 March 2014(Amount`lakh)

\begin{tabular}{|c|c|c|c|c|c|c|c|c|c|}
\hline \multirow{2}{*}{$\begin{array}{l}\text { Sr. } \\
\text { No. }\end{array}$} & \multirow{2}{*}{$\begin{array}{l}\text { Region / } \\
\text { State }\end{array}$} & \multicolumn{2}{|c|}{ Commercial Banks } & \multicolumn{2}{|c|}{$\begin{array}{c}\text { Regional Rural } \\
\text { Bank }\end{array}$} & \multicolumn{2}{|c|}{ Cooperative Bank } & \multicolumn{2}{|c|}{ Total } \\
\hline & & $\begin{array}{l}\text { No. of } \\
\text { SHGs }\end{array}$ & $\begin{array}{l}\text { Saving } \\
\text { Amount }\end{array}$ & $\begin{array}{l}\text { No. of } \\
\text { SHGS }\end{array}$ & $\begin{array}{c}\text { Saving } \\
\text { Amount }\end{array}$ & $\begin{array}{l}\text { No. of } \\
\text { SHGs }\end{array}$ & $\begin{array}{l}\text { Saving } \\
\text { Amount }\end{array}$ & $\begin{array}{l}\text { No. of } \\
\text { SHGs }\end{array}$ & $\begin{array}{l}\text { Saving } \\
\text { Amount }\end{array}$ \\
\hline $\mathbf{A}$ & $\begin{array}{l}\text { Northern } \\
\text { Region }\end{array}$ & 185795 & 22641.01 & 85340 & 5505.92 & 80666 & 6060.38 & 351801 & 34207.31 \\
\hline B & $\begin{array}{c}\text { North } \\
\text { Eastern } \\
\text { Region }\end{array}$ & 115046 & 4911.93 & 147368 & 5765.88 & 29774 & 1489.28 & 292188 & 12167.09 \\
\hline C & $\begin{array}{l}\text { Eastern } \\
\text { Region }\end{array}$ & 701945 & 38370.61 & 428915 & 51919.82 & 243382 & 21724.17 & 1374242 & 112014.60 \\
\hline
\end{tabular}


International Journal of Trend in Scientific Research and Development (IJTSRD) ISSN: 2456-6470

\begin{tabular}{|c|c|c|c|c|c|c|c|c|c|}
\hline $\mathbf{D}$ & $\begin{array}{l}\text { Central } \\
\text { Region }\end{array}$ & 352294 & 26906.84 & 359743 & 18232.35 & 53928 & 6224.38 & 765965 & 51363.57 \\
\hline $\mathbf{E}$ & $\begin{array}{l}\text { Western } \\
\text { Region }\end{array}$ & 512306 & 53142.45 & 135931 & 2800.95 & 297383 & 36724.08 & 945620 & 92667.48 \\
\hline $\mathbf{F}$ & $\begin{array}{c}\text { Southern } \\
\text { Region }\end{array}$ & 2185529 & 221416.40 & 663573 & 45712.57 & 374332 & 50321.87 & 3223434 & 317450.84 \\
\hline & $\begin{array}{l}\text { SRAND } \\
\text { TOTAL }\end{array}$ & 4052915 & 367389.24 & 1820870 & 129937.49 & 1079465 & 122544.16 & 6953250 & 619870.89 \\
\hline \multicolumn{10}{|c|}{ Southern Region } \\
\hline 1 & $\begin{array}{l}\text { Andhra } \\
\text { Pradesh }\end{array}$ & 1025638 & 97455.56 & 395965 & 26154.09 & 26613 & 1919.33 & 1448216 & 125528.98 \\
\hline 2 & Karnataka & 219056 & 21224.22 & 157359 & 14304.26 & 158173 & 27176.84 & 534588 & 62705.32 \\
\hline 3 & Kerala & 306422 & 32282.68 & 34190 & 849.88 & 53585 & 4423.76 & 394197 & 37556.32 \\
\hline 4 & $\begin{array}{l}\text { Tamil } \\
\text { Nadu }\end{array}$ & 618627 & 69400.56 & 75202 & 4377.38 & 132881 & 16595.32 & 826710 & 90373.26 \\
\hline \multirow[t]{2}{*}{5} & Puducherry & 15786 & 1053.38 & 857 & 26.96 & 3080 & 206.62 & 19723 & 1286.96 \\
\hline & TOTAL & 2185529 & 221416.40 & 663573 & 45712.57 & 374332 & $\mathbf{5 0 3 2 1 . 8 7}$ & 3223434 & 317450.84 \\
\hline
\end{tabular}

NABARD intensified the implementation of the programme in the 13 identified priority states. Some of which account for the back or the rural poor viz UP, Orissa, WB, MP, Maharashtra, Gujarat, Rajasthan, Chhattisgarh, Jharkhand, Bihar, Assam, Himachal Pradesh and Uttarkhand. According to the programme spread rapidly in these states indicates a marked shift from its initial localization in the Southern region. In terms of relative shares of different agencies Commercial banks continue to maintain their lead both in terms of number s or SHGs credit linked and loan disbursed through RRB and take the second position of the banks.

Region wise including southern states progress under Micro finance - savings of SHGs shown in the Table 2. As on $31^{\text {st }}$ March 2017, among six regions the first place goes to Southern region. Southern region is with 32, 23,434 SHGs and amounted to Rs 3, 17,450.84 lakhs (51.21\%). The least place goes to North Eastern region with number of SHGs 2,92,188 (4.20\%) and amounted to Rs 12,167.09 lakhs (1.96\%). Where as in Southern region, nearly $44.92 \%$ of total SHGs and $39.54 \%$ of total amount, second and third places goes to Tamil Nadu and Karnataka SHGs 25.65\%, $16.58 \%$ and share of total amount $28.47 \%$ and $19.75 \%$. Accordingly the programme spread rapidly in these states indicating a marked shift in Southern states. The number of SHGs and amount of Non - Southern regions rose from $29 \%$ at the end of 2001 to 53.64.

Table 3: Microfinance - Bank Loans Outstanding against SHGs Region-wise and Southern States as on 31 March 2014(Amount` lakh)

\begin{tabular}{|c|c|c|c|c|c|c|c|c|c|}
\hline \multirow[t]{2}{*}{$\begin{array}{l}\text { Sr. } \\
\text { No. }\end{array}$} & \multirow[t]{2}{*}{$\begin{array}{c}\text { Region / } \\
\text { State }\end{array}$} & \multicolumn{2}{|c|}{ Commercial Banks } & \multicolumn{2}{|c|}{$\begin{array}{l}\text { Regional Rural } \\
\text { Bank }\end{array}$} & \multicolumn{2}{|c|}{ Cooperative Bank } & \multicolumn{2}{|c|}{ Total } \\
\hline & & $\begin{array}{l}\text { No. of } \\
\text { SHGs }\end{array}$ & Loans O/s & $\begin{array}{l}\text { No. of } \\
\text { SHGs }\end{array}$ & $\begin{array}{l}\text { Loans } \\
\mathrm{O} / \mathrm{s}\end{array}$ & & & $\begin{array}{l}\text { No. of } \\
\text { SHGs }\end{array}$ & Loans O/s \\
\hline $\mathbf{A}$ & $\begin{array}{l}\text { Northern } \\
\text { Region }\end{array}$ & 65759 & 49748.24 & 41415 & 17852.18 & 45317 & 13912.91 & 152491 & 81513.33 \\
\hline
\end{tabular}


International Journal of Trend in Scientific Research and Development (IJTSRD) ISSN: 2456-6470

\begin{tabular}{|c|c|c|c|c|c|c|c|c|c|}
\hline B & $\begin{array}{l}\text { North } \\
\text { Eastern } \\
\text { Region }\end{array}$ & 69571 & 38826.10 & 50297 & 22853.97 & 13917 & 5667.72 & 133785 & 67347.79 \\
\hline $\mathrm{C}$ & $\begin{array}{l}\text { Eastern } \\
\text { Region }\end{array}$ & 561579 & 236556.29 & 313118 & 108645.63 & 152873 & 24288.96 & 1027570 & 369490.88 \\
\hline D & $\begin{array}{l}\text { Central } \\
\text { Region }\end{array}$ & 317021 & 157725.85 & 153582 & 77276.47 & 27319 & 11237.28 & 497922 & 246239.60 \\
\hline $\mathbf{E}$ & $\begin{array}{l}\text { Western } \\
\text { Region }\end{array}$ & 331693 & 104591.78 & 38064 & 13365.98 & 87719 & 18990.72 & 457476 & 136948.48 \\
\hline $\mathbf{F}$ & $\begin{array}{l}\text { Southern } \\
\text { Region }\end{array}$ & 1891640 & 1429022.95 & 507504 & 374464.01 & 182968 & 98801.03 & 2582112 & 1902287.99 \\
\hline \multirow{2}{*}{\multicolumn{2}{|c|}{$\begin{array}{l}\text { GRAND } \\
\text { TOTAL } \\
\text { Southern Region }\end{array}$}} & 3237263 & 2016471.21 & 1103980 & 614458.24 & 510113 & 172898.62 & 4851356 & 2803828.07 \\
\hline & & & & & & & & & \\
\hline 1 & $\begin{array}{l}\text { Andhra } \\
\text { Pradesh } \\
\end{array}$ & 1062726 & 868646.25 & 390122 & 290009.69 & 18436 & 15297.68 & 1471284 & 1173953.62 \\
\hline 2 & Karnataka & 168624 & 137616.81 & 75418 & 50527.29 & 56696 & 17386.23 & 300738 & 205530.33 \\
\hline 3 & Kerala & 223741 & 80591.57 & 12042 & 8675.00 & 21977 & 12264.09 & 257760 & 101530.66 \\
\hline 4 & $\begin{array}{l}\text { Tamil } \\
\text { Nadu }\end{array}$ & 424763 & 329292.48 & 29582 & 24911.20 & 84522 & 51738.52 & 538867 & 405942.50 \\
\hline \multirow[t]{2}{*}{5} & Puducherry & 11786 & 12875.84 & 340 & 340.83 & 1337 & 2114.51 & 13463 & 15331.18 \\
\hline & TOTAL & 1891640 & 1429022.95 & 507504 & 374464.01 & 182968 & 98801.03 & 2582112 & 1902287.99 \\
\hline
\end{tabular}

Banks loans outstanding against SHGs region wise region wise and Southern states as on $31^{\text {st }}$ March 2010 was disclosed in Table 2. More than 53\% of SHGs were identified with Southern region and the lowest $2.75 \%$ with North Eastern region. The highest amount of total loans outstanding with Southern region was about $67.85 \%$ and the lowest of $2.4 \%$ with North Eastern region. The second place goes to eastern region in both number of SHGs as well as amount of loans outstanding. While comparing with in Southern region states a major number of SHGs 14, 71,284 (56.98\%) and amount of loan outstanding Rs 868646.25 lakhs (61.72\%). The least place goes to Pondicherry as number of SHGs 13,463 (0.52\%) and amount of Rs 15,331.18 (0.81\%).

Table 4: Bank loans disbursed to SHGs - Agency-wise Position ('in crore)

\begin{tabular}{|c|c|c|c|c|c|c|c|c|}
\hline \multirow[t]{2}{*}{ Agency } & \multirow{2}{*}{$\begin{array}{l}\text { During } \\
\text { the year }\end{array}$} & \multicolumn{4}{|c|}{$\begin{array}{l}\text { Total Loans disbursed by Banks to } \\
\text { SHGs during the year }\end{array}$} & \multirow{2}{*}{$\begin{array}{l}\text { Per SHG } \\
\text { loan } \\
\text { disbursed } \\
\text { (Rupees) }\end{array}$} & \multicolumn{2}{|c|}{$\begin{array}{l}\text { Out of total: Bank } \\
\text { loan disbursed to } \\
\text { SHGs under SGSY }\end{array}$} \\
\hline & & $\begin{array}{l}\text { No of } \\
\text { SHGs }\end{array}$ & $\%$ share & Amount & $\%$ share & & $\begin{array}{l}\text { No of } \\
\text { SHGs }\end{array}$ & Amount \\
\hline $\begin{array}{l}\text { Commercial } \\
\text { Banks } \\
\text { (Public \& } \\
\text { Private } \\
\text { Sector) }\end{array}$ & $\begin{array}{l}2014- \\
15 \\
\% \\
\text { growth }\end{array}$ & $\begin{array}{l}1004587 \\
977521 \\
-2.7\end{array}$ & $\begin{array}{l}62.4 \\
61.6\end{array}$ & $\begin{array}{l}8060.53 \\
9780.19 \\
21.3\end{array}$ & $\begin{array}{l}65.8 \\
67.7\end{array}$ & $\begin{array}{l}80237 \\
100050 \\
24.7\end{array}$ & $\begin{array}{l}133117 \\
157560 \\
18.4\end{array}$ & $\begin{array}{l}1102.38 \\
1215.50 \\
10.3\end{array}$ \\
\hline $\begin{array}{l}\text { Regional } \\
\text { Rural Banks }\end{array}$ & $\begin{array}{l}2014- \\
15 \\
\% \\
\text { growth }\end{array}$ & $\begin{array}{l}405569 \\
376797 \\
-7.1\end{array}$ & $\begin{array}{l}25.2 \\
23.7\end{array}$ & $\begin{array}{l}3193.49 \\
3333.20 \\
4.4\end{array}$ & $\begin{array}{l}26.1 \\
23.1\end{array}$ & $\begin{array}{l}78741 \\
88461 \\
12.3\end{array}$ & $\begin{array}{l}81662 \\
67531 \\
-17.3\end{array}$ & $\begin{array}{l}655.27 \\
682.41 \\
4.1\end{array}$ \\
\hline $\begin{array}{l}\text { Cooperative } \\
\text { Banks }\end{array}$ & $\begin{array}{l}2014- \\
15\end{array}$ & $\begin{array}{l}199430 \\
232504\end{array}$ & $\begin{array}{l}12.4 \\
14.7\end{array}$ & $\begin{array}{l}999.49 \\
1339.92\end{array}$ & $\begin{array}{l}8.2 \\
9.3\end{array}$ & $\begin{array}{l}50117 \\
57629\end{array}$ & $\begin{array}{l}49874 \\
42312\end{array}$ & $\begin{array}{l}257.57 \\
300.09\end{array}$ \\
\hline
\end{tabular}


International Journal of Trend in Scientific Research and Development (IJTSRD) ISSN: 2456-6470

\begin{tabular}{|c|c|c|c|c|c|c|c|c|}
\hline & $\begin{array}{l}\% \\
\text { growth }\end{array}$ & 16.6 & & 34.1 & & 15.0 & -15.2 & 16.5 \\
\hline Total & $\begin{array}{l}2014- \\
15 \\
\% \\
\text { growth }\end{array}$ & $\begin{array}{l}1609586 \\
1586822 \\
-1.4\end{array}$ & $\begin{array}{l}100.0 \\
100.0\end{array}$ & $\begin{array}{l}12253.51 \\
14453.30 \\
17.9\end{array}$ & $\begin{array}{l}100.0 \\
100.0\end{array}$ & $\begin{array}{l}76128 \\
91083 \\
19.6\end{array}$ & $\begin{array}{l}264653 \\
267403 \\
1.0\end{array}$ & $\begin{array}{l}2015.22 \\
2198.00 \\
9.1\end{array}$ \\
\hline
\end{tabular}

Table 5: Bank loan outstanding against SHGs - Agency-wise Position (’ in crore)

\begin{tabular}{|c|c|c|c|c|c|c|c|c|}
\hline \multirow[t]{2}{*}{ Agency } & \multirow[t]{2}{*}{$\begin{array}{l}\text { Position } \\
\text { as on }\end{array}$} & \multicolumn{4}{|c|}{$\begin{array}{l}\text { Total Bank Loans outstanding against } \\
\text { SHGs }\end{array}$} & \multirow[t]{2}{*}{$\begin{array}{l}\text { Per SHG } \\
\text { bank } \\
\text { loan o/s } \\
\text { (Rupees) }\end{array}$} & \multicolumn{2}{|c|}{$\begin{array}{l}\text { Out of total: Bank } \\
\text { loan outstanding } \\
\text { against SHGS } \\
\text { under SGSY }\end{array}$} \\
\hline & & $\begin{array}{l}\text { No of } \\
\text { SHGs }\end{array}$ & $\%$ share & Amount & $\%$ share & & $\begin{array}{l}\text { No of } \\
\text { SHGs }\end{array}$ & Amount \\
\hline $\begin{array}{l}\text { Commercial } \\
\text { Banks } \\
\text { (Public \& } \\
\text { Private } \\
\text { Sector) } \\
\end{array}$ & $\begin{array}{l}2014- \\
15 \\
\% \\
\text { growth }\end{array}$ & $\begin{array}{l}2831374 \\
3237263 \\
14.3\end{array}$ & $\begin{array}{l}61.7 \\
66.7\end{array}$ & $\begin{array}{l}16149.43 \\
20164.71 \\
24.9\end{array}$ & $\begin{array}{l}71.2 \\
71.9\end{array}$ & $\begin{array}{l}57037 \\
62289 \\
9.2\end{array}$ & $\begin{array}{l}645145 \\
798304 \\
23.7\end{array}$ & $\begin{array}{l}3961.53 \\
4072.03 \\
2.7\end{array}$ \\
\hline $\begin{array}{l}\text { Regional } \\
\text { Rural Banks }\end{array}$ & $\begin{array}{l}2014- \\
15 \\
\% \\
\text { growth }\end{array}$ & $\begin{array}{l}977834 \\
1103980 \\
14.3\end{array}$ & $\begin{array}{l}23.1 \\
22.8\end{array}$ & $\begin{array}{l}5224.4 \\
6144.58 \\
17.6\end{array}$ & $\begin{array}{l}23.0 \\
21.9\end{array}$ & $\begin{array}{l}53428 \\
55658 \\
4.2\end{array}$ & $\begin{array}{l}258890 \\
368795 \\
42.4\end{array}$ & $\begin{array}{l}1508.10 \\
1725.94 \\
14.4\end{array}$ \\
\hline $\begin{array}{l}\text { Cooperative } \\
\text { Banks }\end{array}$ & $\begin{array}{l}2014- \\
15 \\
\% \\
\text { growth }\end{array}$ & $\begin{array}{l}415130 \\
510113 \\
22.9\end{array}$ & $\begin{array}{l}9.8 \\
10.5\end{array}$ & $\begin{array}{l}1306.00 \\
1728.99 \\
32.4\end{array}$ & $\begin{array}{l}5.8 \\
6.2\end{array}$ & $\begin{array}{l}31460 \\
33894 \\
7.7\end{array}$ & $\begin{array}{l}72852 \\
78295 \\
7.5\end{array}$ & $\begin{array}{l}392.09 \\
453.11 \\
15.6\end{array}$ \\
\hline Total & $\begin{array}{l}2014- \\
15 \\
\% \\
\text { growth }\end{array}$ & $\begin{array}{l}4224338 \\
4851356 \\
14.8\end{array}$ & $\begin{array}{l}100.0 \\
100.0\end{array}$ & $\begin{array}{l}22679.85 \\
28038.28 \\
23.6\end{array}$ & $\begin{array}{l}100.0 \\
100.0\end{array}$ & $\begin{array}{l}53689 \\
57795 \\
7.6\end{array}$ & $\begin{array}{l}976887 \\
1245394 \\
27.5\end{array}$ & $\begin{array}{l}5861.72 \\
6251.07 \\
6.6\end{array}$ \\
\hline
\end{tabular}

\section{Coverage of Women SHGs}

The details of total number of women SHGs saving linked, credit linked and loans outstanding for the last two years are given in table 6

Table 6: Position of Women SHGs ( in crore)

\begin{tabular}{|c|c|c|c|c|c|c|c|}
\hline \multirow[t]{2}{*}{ Particulars } & \multirow[t]{2}{*}{ Year } & \multicolumn{2}{|c|}{ Total SHGs } & \multicolumn{2}{|c|}{$\begin{array}{l}\text { Exclusive } \\
\text { sHGs }\end{array}$} & \multicolumn{2}{|c|}{$\begin{array}{l}\% \text { age of women } \\
\text { SHGs to total SHGs }\end{array}$} \\
\hline & & No. & Amt & No. & Amt & No & Amt. \\
\hline \multirow{2}{*}{$\begin{array}{l}\text { Saving } \\
\text { linked } \\
\text { SHGs }\end{array}$} & 31.03 .2009 & 6121147 & 5545.62 & 4863921 & 4434.03 & 79.5 & 80.0 \\
\hline & 31.03 .2010 & 6953250 & 6198.71 & 5310436 & 4498.66 & 76.4 & 72.6 \\
\hline \multirow{2}{*}{$\begin{array}{l}\text { Loans } \\
\text { disbursed }\end{array}$} & 2008-09 & 1609586 & 12253.51 & 1374579 & 10527.38 & 85.4 & 85.9 \\
\hline & 2009-10 & 1586822 & 14453.30 & 1294476 & 12429.37 & 81.6 & 86.0 \\
\hline \multirow{2}{*}{$\begin{array}{l}\text { Loans } \\
\text { Outstanding }\end{array}$} & 31.03 .2009 & 4224338 & 22679.84 & 3277355 & 18583.54 & 77.6 & 81.9 \\
\hline & 31.03 .2010 & 4851356 & 28038.28 & 3897797 & 23030.36 & 80.3 & 82.1 \\
\hline
\end{tabular}

Micro Finance Development and Equity Fund 
To strengthen the efforts of NABARD towards promotional support for micro finance, the Government of India in the Union Budget for 2016-17 had further increased the corpus of Micro Finance Development and Equity Fund (MFDEF) to ' 600 crore. Recognising the need for upscaling the microFinance interventions in the country, the Hon'ble Union Finance Minister, while presenting the budget for the year 2000-01, had created Micro Finance Development Fund (MFDF) with an initial contribution of ' 100 crore, to be funded by Reserve Bank of India, NABARD and commercial Banks in the ratio of 40:40:20. In the Union Budget for 200506, the Government of India had decided to redesignate the mFDF into $\mathrm{mFDEF}$ and raised its corpus from ' 100 crore to 200 crore. The mFDEF is managed and administered by NABARD under the guidance of an mFDEF Advisory Board. The objective of mFDEF is to facilitate and support the orderly growth of the microfinance sector through diverse modalities for enlarging the flow of financial services to the poor, particularly for women and vulnerable sections of society consistent with sustainability.

\section{CONCLUSION}

The Task Force on supportive policy and regulatory Frame work for Microfinance constituted by NABARD defined microfinance as "the provision of thrift, saving, credit and financial services and products of very small amount to the poor in rural, semi-urban and urban areas for enabling them to raise their income levels and improving their standard of living." Since the SHGs were able to mobilize savings from the poor who were not expected to have any savings and could also recycle effectively the pooled saving among the members, they succeeded in performing banking services to their members may be in a primitive way but in a manner which was cost effective, simple, flexible at the door steps of the members and above all without defaults in repayment by borrowings which is well managed by the poor illiterate women. Women, who number 495.7 million according to 2001 census, represent $48.3 \%$ and 933 females per thousand males. It reveals that the decline of women number in total population of the country. Moreover nearly $1.5 \%$ of women have their own property. About $11 \%$ women are in employment and mere $5 \%$ have been participating in business. The present paper is an attempt to study the Empowerment of women through SHG's in Andhra Pradesh.

\section{REFERENCES}

1) Fisher, T. and Sriman, M.S. 2002, Beyond Micro - Credit, putting development back into Micro Finance, Vistaar Publications, New Delhi.

2) Karmakar, K.G. 1999. Rural credit and Self Help Groups, Micro Finance needs and concepts in India, Sage Publications.

3) NABARD Annual Reports 2014 - 15 to 2016 17.

4) NABARD 2009 - 10 Progress of SHGs Bank Linkage in India, Mumbai.

5) Pillai, B.V. and V. Hari Kumar 2006 Self Help Groups in Kerala, Kurukshetra, July.

6) RBI 2009 -16 Annual Report, Mumbai.

7) RBI 2009 -16 Report on Trend and Progress on Banking in India, Mumbai.

8) B.Jayaraman, 2014 Micro Finance, Retrospects, occasional paper - 20 NABARD.

9) Jana Madan Mohan, 2011, “ Corporate Financial Inclusion Plan in India - An inclusive Growth Approach - An Empirical Study" The Management Accountant, October p, 897.

10) Report of the Committee on Financial Inclusion, Chairman - Dr. C.Rangarajan, Jan ,2008. 